This is an original manuscript / preprint of an article published by Taylor \&

Francis in Advanced Robotics on 22 Sep 2020, available online:

https://www.tandfonline.com/doi/full/10.1080/01691864.2020.1822913.

\title{
Design and performance characterization of a soft robot hand with fingertip haptic feedback for teleoperation
}

\author{
Min $\mathrm{Li}^{\mathrm{a}}{ }^{\mathrm{d} *}$, Yueyan Zhuo ${ }^{\mathrm{a}}$, Jiazhou $\mathrm{Chen}^{\mathrm{a}}$, Bo He ${ }^{\mathrm{a}}$, Guanghua $\mathrm{Xu}^{\mathrm{a}, \mathrm{c}}$, Jun \\ Xie $^{\mathrm{a}}$, Xingang Zhao ${ }^{\mathrm{d}}$, Wei Yao ${ }^{\mathrm{b} *}$ \\ a Department of Mechanical Engineering, Xi'an Jiaotong University, Xi'an 710049,
}

China

b Department of Biomedical Engineering, University of Strathclyde, Glasgow G4 ONW, $U K$

c State Key Laboratory for Manufacturing Systems Engineering, Xi'an Jiaotong University, Xi'an 710054, China

\author{
d State Key Laboratory of Robotics, Shenyang Institute of Automation, Chinese \\ Academy of Sciences, Liaoning 110016, China
}

Provide full correspondence details here including e-mail for the * corresponding author
Abstract: A soft robot hand with fingertip haptic feedback for teleoperation is proposed to perform complex tasks and ensure safe and friendly human-machine interaction. This robot hand can perform finger flexion/extension and abduction/adduction motions. A data glove is used to collect the hand joint angle data of the operator. Flexion sensors are embedded in the soft robot hand to monitor the bending angles of the actuators. Pressure sensors on the fingertips of the robot hand collect contact force data, and haptic feedback actuators located on the fingertips of the operator display the contact force to the operator.
Characterization tests and teleoperation performance tests involving human participants are performed to prove the feasibility of the soft robot hand. The soft robot hand prototype satisfies the output force requirements and can meet $96.86 \%$ of the design requirements of the joint angles. The soft robot hand can be teleoperated to perform nine commonly used motions in daily operational tasks. The success rates of fingertip force discrimination, grasp, and pinch ability experiment are $100 \%, 95.00 \%$, and $98.33 \%$, respectively. The results of the experiment suggest that the soft robot hand with fingertip haptic feedback can perform complex tasks in teleoperation. 
This is an original manuscript / preprint of an article published by Taylor \& Francis in Advanced Robotics on 22 Sep 2020, available online: https://www.tandfonline.com/doi/full/10.1080/01691864.2020.1822913.

Keywords: soft robot hand; teleoperation; fingertip haptic feedback; pose sensing Subject classification codes: include these here if the journal requires them

\section{Introduction}

Robot hands comprise a type of devices that can mimic hand movements by using functions for grasping, carrying objects, and operating tools. Complex tasks may be inconvenient or risky to perform at the scene and may be difficult for robots alone to handle. In such cases, highly dexterous robot hands teleoperated by humans at a safe distance need to be employed. High dexterity of the robot hand and accurate remote control capability are two requirements for robot hands in such applications.

Complex tasks require high dexterity of the robot hand. The complexity and intelligence of biological systems have long prompted engineering efforts [1]. Over the past decades, research on biomimetic robotic hands has reached encouraging achievements. Dexterous hands are a type of advanced robotic hands that mimic human hand movements. They generally have more than three fingers, and each finger has more than three degrees of freedom (DOFs). The Utah/MIT hand and Stanford/JPL hand developed in the 1980s are milestones in the history of dexterous hands [2,3]. Other notable examples include the DLR Hand, Gifu Hand, ACT Hand, and Shadow Dexterous Hand [4-8]. However, a dexterous hand usually consists of rigid phalanges and joints for accurate operation and sufficient grasping force, resulting in limited contact points and uneven distribution of contact forces.

Teleoperation applications such as grasping of fragile objects with complex shapes, recusing injured people, and taking care of patients with infectious diseases require the robot hand to offer certain flexibility and adaptability to ensure safe and friendly human-machine interaction. As another type of robot hands, soft robot hands 
This is an original manuscript / preprint of an article published by Taylor \& Francis in Advanced Robotics on 22 Sep 2020, available online: https://www.tandfonline.com/doi/full/10.1080/01691864.2020.1822913.

can use soft joints for passive rotation to adapt to object shape. They are more suitable for the aforementioned teleoperation applications than robot hands with rigid phalanges and joints. Soft joints are typically constructed with spring components $[9,10]$, soft tendon systems [11-14], or pneumatic actuators [15-17]. The softness of robot hands can improve dexterity and provide impact reduction at contact, safe interaction with humans and objects, and high adaptation capacity to object shapes [18]. They can also perform various grasp motions with few actuators because of deformability, which further reduces costs and complexity. However, the deformability of soft robot hands has some negative effects on controllability, control accuracy and durability [19]. To deal with the durability problem of soft actuators, Wang et al. [19] resorted to a fabric compositing method creating a fabric/rubber composite that increased the strength and durability of the molded rubber. Yap et al. [20] developed 3D-printed soft pneumatic actuators through fused deposition modeling technology using NinjaFlex (NinjaTek, PA). Their experimental results showed that the durability of the actuators created through this method was better than the 3D-printed actuators developed by Peele et al. using Projection Stereolithography [21].

The controllability and control accuracy of soft robot hands can be improved in three aspects. Sensing the pose of robot hands in real time can easily result in accurate remote control. However, the deformability or flexibility of soft robotic hands impedes pose estimation. Information from conventional sensors, such as encoders, provides no sufficient information when the fingertip surface is deformable. Instead of encoders, soft or flexible sensors such as soft flexion sensors [22,23], cable sensors [24], optoelectronic sensors [25], and microfluidic soft sensors [26] are preferable for detecting the shape of soft robots. 
This is an original manuscript / preprint of an article published by Taylor \& Francis in Advanced Robotics on 22 Sep 2020, available online: https://www.tandfonline.com/doi/full/10.1080/01691864.2020.1822913.

Another method to improve the capability for accurate teleoperation of the robot hand is to enhance the degree of "immersion" of the operator when performing a teleoperational task by introducing intuitive teleoperation techniques [27,28].

Electromyography (EMG) can capture human hand movements and control robot hands [29]. However, use of EMG signals to decode complex finger movements remains inconclusive. Therefore, EMG-based teleoperation can only support simple grasps. Data gloves can also be used as the input device to provide intuitive control for teleoperation [30]. Detailed finger movements can be captured in real time by using data gloves.

Employing haptic feedback to convey the information of the contact object can also enhance human-robot interaction during teleoperational tasks and improve the accurate teleoperation capability of the robot hand [27,28]. Wearable haptic systems for the fingertip and the hand come in three types: grounded haptics, exoskeletons, and fingertip devices. Although they can provide contact force higher than that of fingertip devices, grounded haptics and exoskeletons are often heavy and cumbersome, limiting their practical application [28]. Fingertip devices are more lightweight than grounded haptics and exoskeletons and can be easily integrated into other systems [28].

In the present study, a 3D-printed soft robot hand with haptic feedback at the fingertip is proposed for teleoperation to help operator grasp or operate objects flexibly, when robot hands are required to ensure safe interaction and high adaptation capacity to the shape of objects under circumstances such as grasping fragile items, rescuing injured people, and interacting with patients remotely. The main advantages of this soft robot hand include the following: (1) the proposed soft robot hand can perform both flexion/extension and abduction/adduction finger motions; (2) the fingertip contact forces are fed back with small and lightweight haptic feedback actuators to the hand of the operator. Section 2 describes the design, fabrication, and integration of the soft robot 
This is an original manuscript / preprint of an article published by Taylor \& Francis in Advanced Robotics on 22 Sep 2020, available online: https://www.tandfonline.com/doi/full/10.1080/01691864.2020.1822913.

hand. In Section 3, experiments are performed to characterize the functionality and the teleoperation performance of the proposed soft robot hand. Conclusions are provided in Section 4 .

\section{Methodology}

\subsection{Overall design}

Figure 1 presents the diagrammatic sketch of the proposed soft robot hand with fingertip haptic feedback for teleoperation. The soft robot hand can grasp, carry objects, or operate tools to perform tasks. The hand movements of the operator are captured using a data glove. The soft robot hand is controlled to follow the hand movements. It is embedded with flexion sensors to monitor the bending angles of the actuators. Pressure sensors on the fingertips of the soft robot hand collect contact force data during the process. Haptic feedback actuators located on the fingertips of the operator display the contact force to the operator.

\subsection{Hand design}

To perform complex tasks, the soft robot hand needs to generate an adequate range of motion (ROM) and sufficient force to grasp, carry objects, or operate tools. The human thumb has an interphalangeal joint (IPJ), a metacarpophalangeal joint (MCPJ), and a carpometacarpal joint (CMCJ); meanwhile, each of the other four human fingers has a distal interphalangeal joint (DIPJ), a proximal interphalangeal joint (PIPJ), and an MCPJ. In the current study, the soft robot hand contains five fingers with the DOFs of the flexion/extension motions at DIPJ, PIPJ, IPJ, MCPJ, and CMCJ and the DOFs of the abduction/adduction motion between each pair of adjacent fingers at MCPJ. 
This is an original manuscript / preprint of an article published by Taylor \& Francis in Advanced Robotics on 22 Sep 2020, available online: https://www.tandfonline.com/doi/full/10.1080/01691864.2020.1822913.

The data (Table 1) obtained by Lee and Jung on the flexion ROM of finger joints [31] were used as the design requirements of our soft robot hand. To determine the abduction/adduction ROM of finger joints, we measured the motion ranges of 20 subjects, consisting of 18 healthy males and 2 healthy females, during abduction/adduction. Average maximum angles were then calculated (Table 1).

Nine commonly used motions in operational tasks-light tool grasp, medium wrap grasp, fixed hook, spherical grasp, power disk grasp, tripod grasp, palmar grasp, lateral pinch, and precision tip pinch [32-34]—-were chosen as the target grasp and operate motions of the proposed soft robot hand. According to Polygerinos et al. [35], the force generated by one finger should be greater than $7.3 \mathrm{~N}$ to perform motions in grasping and manipulation tasks when handling objects that weigh less than $1.5 \mathrm{~kg}$.

The proposed soft robot hand consisted of bending actuators and abduction actuators. The 3D models of the bending and abduction actuators are presented in Figure 2. The bending actuator of the thumb had two independently controlled air channels. The IPJ could perform independent motions without affecting the MCPJ motion. The CMCJ actuator also had one independently controlled air channel. For other fingers, the DIPJ and PIPJ shared one air channel because the motion of human DIPJ is associated with the flexion angle of the PIPJ [36]. The MCPJ had one independently controlled air channel. The bending actuators of the fingers consisted of inflexible parts for the phalanges and inb parts for the joints. The flexible parts adopted a single-air cavity structure for flexion deformation, whereas the inflexible parts connected the soft parts and limited deformation. Both the flexible and inflexible parts used the same material. The characteristics of flexible and inflexible came from the differences in structures rather than in materials. 
This is an original manuscript / preprint of an article published by Taylor \& Francis in Advanced Robotics on 22 Sep 2020, available online: https://www.tandfonline.com/doi/full/10.1080/01691864.2020.1822913.

When the air chamber was filled with pressurized air, the actuator would bend. When the air pressure was released, the actuator returned to its original state because of material elasticity. Consequently, the soft robot hand could perform complex tasks by the combination of flexion/extension and abduction/adduction motions of those actuators.

\subsection{Pose sensing and control for hand teleoperation}

To perform teleoperation tasks, the soft robot hand should exhibit the ability to identify the intention of the operator. To achieve closed-loop control, the movements of the soft robot hand should be monitored.

To detect the intention of the operator, a data glove (WiseGlove14, Beijing Xintian Shijing Technology, China) was used to collect the motion data of the fingers. This data glove, which contained 14 optical fiber sensors, exhibited a dynamic accuracy of $0.2^{\circ}$ and a data refresh rate of $100 \mathrm{~Hz}$. The flexion/extension and abduction/adduction angles of the robot hand were calculated based on the sensed data, transferred into analog signals and then sent to an analog input/output module (JY-

DAM-AIAO, Beijing Elit Gathering Electron, China) to control the pressure regulators (IVT0030-2BL, SMC, Japan) that connect the air channels of the soft robot. For pose sensing, flexion sensors (RFP, Yubo Intelligent Technology, China) were employed to collect the data of the flexion/extension angles of the robot hand. A closed-loop PID control was then used for the flexion/extension angles of the robot hand.

\subsection{Fingertip contact force sensing and haptic feedback}

Haptic feedback plays an important role in improving the performance of a teleoperation system and reducing task completion time [37-39]. The operator should 
This is an original manuscript / preprint of an article published by Taylor \& Francis in Advanced Robotics on 22 Sep 2020, available online: https://www.tandfonline.com/doi/full/10.1080/01691864.2020.1822913.

receive sufficient contact information between the robot hand and the environment to feel physically present at the remote site [40]. To enhance the "immersion" of the operator when performing tasks, fingertip haptic feedback was provided in the proposed system. Fingertip pressure sensors (RP-C10-ST-LF, measurement force range: 50-2000 g, Waaax, China) were mounted on the fingertips of the soft robot hand to monitor the contact force information.

People usually interact with an object by touching, licking, pinching, and colliding with their fingertips. The haptic forces generated by these motions vary; however, when these motions are performed by the fingertips, the main force received by the finger is the normal force. The proposed fingertip wearable haptic feedback actuator only provided normal contact force to the fingertip to reduce the complexity of the mechanism and the control system. Fingertip haptic devices usually have a maximum haptic contact force ranging from $0.60 \mathrm{~N}$ to $6.00 \mathrm{~N}$ [41-44]. The target maximum force generated by our haptic feedback actuator was set to $6.00 \mathrm{~N}$.

The haptic feedback actuator was composed of a contact surface and an air chamber (Figure 3). The working surface was similar to an ellipse. When pressurized air filled the actuator chamber, the contact surface was deformed to generate normal force to the fingertip.

Pressure sensors were mounted on the fingertips of the soft robot hand to collect pressure information on the fingertip and transmit data to the analog input/output module. The analog input/output module was linked to the pressure regulators to control the pressure in the air chambers of the haptic feedback actuators. The haptic feedback actuators were fastened to the fingertips of the operator by using straps to generate corresponding normal forces to the fingertips. 
This is an original manuscript / preprint of an article published by Taylor \& Francis in Advanced Robotics on 22 Sep 2020, available online: https://www.tandfonline.com/doi/full/10.1080/01691864.2020.1822913.

\subsection{Fabrication and integration}

The bending, abduction, and haptic feedback actuators in the soft robot hand were 3Dprinted using a LulzBot TAZ 6 Aerostruder printer with a soft material (NinjaFlex 85A TPU). The slicer software Cura-LulzBot 3.2.21 was used. The flexible parts and the inflexible parts that belonged to the same actuator were 3D-printed in one piece using the same material. No support materials were required to print the chambers. When printing the parts above the chambers, the material would sag a little for the first few layers without affecting the function of the actuators. The printing parameters were adjusted as shown in Table 2 .

Bending and abduction actuators were mounted to a polylactic acid (PLA) palm (Figure 4). Silicone rubber (Dragon Skin 10 Medium, Smooth-On, USA) was used to cover the surface of the bending actuators and thus fix the flexion sensors and pressure sensors on the fingers of the soft robot hand. First, the finger actuator and the sensors were placed in a 3D-printed mold. Then, liquid silicone rubber was poured into the gap between the actuator and the mold to make the layer covering the finger actuator and the sensors. At last, air tubes were glued to the actuators by using silicone sealant (RTV108, Momentive Performance Materials, USA).

The data glove sent the data of the hand movements to a laptop computer (Windows 10, Intel (R) Core (TM) i7-8750H CPU@ 2.2 GHz, 8GB RAM, and 64-bit operating system). Two analog input/output modules were employed to collect data from the sensors and send analog signals to the pressure regulators. Pressurized air was provided by an air compressor (U-STAR601, U-STAR, China). 
This is an original manuscript / preprint of an article published by Taylor \& Francis in Advanced Robotics on 22 Sep 2020, available online: https://www.tandfonline.com/doi/full/10.1080/01691864.2020.1822913.

\section{Experiments and results}

\subsection{Robot hand characterization}

Characterization tests were performed regarding the weight, dimensions, ROM, flexion force, abduction force, and fingertip haptic feedback force of the soft robot hand. The details of the characterization tests are presented in the following sections.

\subsubsection{Weight and dimensions}

The dimension of the soft robot hand was $238 \mathrm{~mm} \times 167 \mathrm{~mm} \times 41 \mathrm{~mm}$. The soft robot hand was slightly larger than the hand of an adult man (Figure 4(b)). The soft robot hand, including the PLA palm, flexion, and abduction actuators, weighed $435.92 \mathrm{~g}$. A haptic feedback actuator measured about $20 \mathrm{~mm} \times 16 \mathrm{~mm} \times 10 \mathrm{~mm}$ and weighed 2.30 g.

\subsubsection{Range of Motion}

In the ROM test, the flexion and abduction actuators were pressurized to perform flexion and abduction, and the flexion and abduction angles generated using different amounts of pressure were recorded using a VICON motion capture system. Markers were attached to the soft robot hand (Figure 5). The test was repeated five times.

The average flexion and abduction angles of soft hand fingers are shown in Figure 6. Table 3 presents a comparison between the ROM of the soft robot hand and the design requirements. The soft robot hand achieves $96.02 \%$ of the maximum finger joint flexion angles and $100 \%$ of the maximum abduction angles. This result proves that the soft robot hand can perform motions commonly used in tasks. 
This is an original manuscript / preprint of an article published by Taylor \& Francis in Advanced Robotics on 22 Sep 2020, available online: https://www.tandfonline.com/doi/full/10.1080/01691864.2020.1822913.

\subsubsection{Pose sensing and fingertip contact force sensing}

In our robot hand, flexion sensors were embedded to measure the flexion/extension angles of the robot hand. An evaluation test was conducted to confirm whether the deformation of the robot hand could be expressed by the sensor output. During the test, the flexion actuators were pressurized from 0 to $300 \mathrm{kPa}$ with a $50 \mathrm{kPa}$ interval to perform flexion. Each test was repeated five times. As shown in Figure 7, the outputs from the flexion sensors were compared to the outputs from a VICON motion capture system. The root-mean-square error was $2.04^{\circ}$.

In our robot hand, pressure sensors were mounted on the fingertips to monitor the contact force information. The pressure sensors were calibrated using a force sensor (Nano17, SI-12-0.12, resolution of $0.003 \mathrm{~N}$ with a 16-bit data acquisition card, ATI Industrial Automation, USA). During the process, the force sensor and the fingertip with the pressure sensor were directly contacted with each other. A normal force ranging from 0 to $5 \mathrm{~N}$ with an interval of $1 \mathrm{~N}$ was applied to the fingertip of the soft robot hand by using a clamp. The test for each finger was repeated three times. The relationship between the outputs of the pressure sensor and the force sensor was then described using a quadratic polynomial regression model. The determining coefficients $\left(\mathrm{R}^{2}\right)$ of the regression models of the five fingers were 0.9686 (thumb), 0.9583 (index finger), 0.8767 (middle finger), 0.9550 (ring finger), and 0.9097 (pinky).

An evaluation test was conducted to investigate the force sensor outputs when the actuators in those robot fingers were pressurized and no external contact force was applied. Each finger was pressurized to perform a flexion motion until the maximum flexion range was reached. The pressure sensor output was recorded during the test. Each test was repeated five times. The range of the sensor outputs due to the bending 
This is an original manuscript / preprint of an article published by Taylor \&

Francis in Advanced Robotics on 22 Sep 2020, available online:

https://www.tandfonline.com/doi/full/10.1080/01691864.2020.1822913.

motion of the robot hand was from 0 to $0.10 \mathrm{~N}$. The average output of the sensors due to the bending motion was $0.02 \mathrm{~N}$ with a standard deviation of $0.04 \mathrm{~N}$.

\subsubsection{Flexion force}

The output force of the robot hand fingers was examined. A finger of the soft robot hand was pressurized to $300 \mathrm{kPa}$ to grasp a PLA cylinder with a diameter of $15 \mathrm{~mm}$. The soft robot hand was fixed by a clamp (Figure 8(a)). The PLA cylinder was pulled up by a ball screw to unfold the finger until the actuator released the cylinder. A force sensor (BBTGTJL-1, measurement range of $0-50 \mathrm{~kg}$ and precision of $0.5 \mathrm{~g}$, BBTG, USA) was used to collect the pull force, and the data were recorded using a data acquisition card (NI USB-6210, NI, USA). Each test was repeated three times.

The forces required to unfold the fingers and the friction force that could be generated by each finger are listed in Table 4 . All forces were greater than our design requirement of $7.3 \mathrm{~N}$, considering that the objects in most grasping and manipulation tasks in daily life usually weigh less than $1.5 \mathrm{~kg}$ [45]. The soft robot hand with five fingers could generate a total force of $73.34 \mathrm{~N}$ to lift an object. The friction force of each finger was calculated considering the mean friction coefficient of the NinjaFlex 85A TPU (0.61 [46]).

\subsubsection{Abduction force}

We examined the output force of the abduction actuators. In the abduction force test, the soft robot hand fingers and the force sensor were fixed using a PLA structure (Nano17, SI-12-0.12, resolution of $0.003 \mathrm{~N}$ with a 16-bit data acquisition card, ATI Industrial Automation, USA) (Figure 8(b)). The abduction actuator was pressurized to $300 \mathrm{kPa}$, and the abduction force was recorded using a data acquisition card (NI USB-6210, NI, USA). Each test was repeated three times. The average maximum force of the abduction 
This is an original manuscript / preprint of an article published by Taylor \& Francis in Advanced Robotics on 22 Sep 2020, available online: https://www.tandfonline.com/doi/full/10.1080/01691864.2020.1822913. actuator between the thumb and the index finger was $23.77 \mathrm{~N}$ with a standard deviation of $0.10 \mathrm{~N}$, and the average maximum force of other abduction actuators was $8.6 \mathrm{~N}$ with a standard deviation of $0.22 \mathrm{~N}$.

\subsubsection{Fingertip haptic feedback force}

The output force of the fingertip haptic feedback actuators was measured using the experimental set-up shown in Figure 9(a). The actuator was fixed at one side of a guide rail. A force sensor (Nano17, SI-12-0.12, resolution of $0.003 \mathrm{~N}$ with a 16-bit data acquisition card, ATI Industrial Automation, USA) was fixed to the sliding block on the guide rail. Before the test, the force sensor was moved to just contact the haptic feedback actuator. The actuator was pressurized from 0 to $100 \mathrm{kPa}$ and then released to $0 \mathrm{kPa}$. The contact forces were recorded continuously during the process with a sampling frequency of $1000 \mathrm{~Hz}$. This test was repeated five times.

The output force data exhibited very good repeatability (Figure 9 (b)). The relationship between the contact force and the applied air pressure was then described using a quadratic polynomial regression model. The determining coefficient $\left(\mathrm{R}^{2}\right)$ of the regression models of the actuator was 0.9434 . The contact force with $100 \mathrm{kPa}$ air pressure was $7.11 \mathrm{~N}$, which was greater than the design requirement $6.00 \mathrm{~N}$. The result demonstrated that the haptic feedback actuator could provide sufficient fingertip haptic force to render the haptic information when performing teleoperation tasks.

\subsection{Teleoperation performance}

\subsubsection{Experimental protocols}

To validate the teleoperation performance of the proposed soft robot hand, motion test, fingertip force discrimination, grasp ability, and pinch ability were conducted. The fingertip force discrimination, grasp ability, and pinch ability 
This is an original manuscript / preprint of an article published by Taylor \& Francis in Advanced Robotics on 22 Sep 2020, available online: https://www.tandfonline.com/doi/full/10.1080/01691864.2020.1822913.

experiments included 12 participants (10 males and 2 females), and each experiment was repeated five times. The study was approved by the Institutional Review Board of Xi'an Jiaotong University. All subjects signed an informed consent prior to the start of the experiments.

In the motion test experiment, we evaluated the prototype of the soft robot hand with fingertip haptic feedback for teleoperation by controlling the prototype to perform nine motions that are commonly used in operation tasks, including light tool grasp, medium wrap grasp, fixed hook, spherical grasp, power disk grasp, tripod grasp, palmar grasp, lateral pinch, and precision tip pinch.

In the fingertip force discrimination experiment, force was applied to the index fingertip of the soft robot hand by using a clamp (Figure 10(a)). The output signals of the pressure sensor were first converted to force data via the polynomial regression model acquired in the calibration experiment described in Section 3.1.3. The force values were then used to determine the target values of the internal air pressure of the haptic device by using the polynomial regression model between the output force and applied air pressure acquired in the experiment described in Section 3.1.6. The participant wore the fingertip haptic feedback actuators and were blindfolded. The force measured by the embedded pressure sensor was displayed to the researcher during the test. At the beginning of the experiment, the initial force generated by the clamp on the robot hand was set to $2 \mathrm{~N}$. During the experiment, the researcher randomly increased or decreased the pressure on the fingertip of the soft robot hand by tightening or untightening the clamp. The force changing range was between 0 and $5 \mathrm{~N}$. The participant was asked whether the force had increased or decreased. If the increase or decrease in force perceived by the subject was consistent with the ground truth, the trial was recorded as a success; otherwise, the trial was recorded as a failure. 
This is an original manuscript / preprint of an article published by Taylor \& Francis in Advanced Robotics on 22 Sep 2020, available online: https://www.tandfonline.com/doi/full/10.1080/01691864.2020.1822913.

In the grasp ability experiment, the participant was asked to control the soft robot hand to grasp the PLA cylinder with a diameter of $60 \mathrm{~mm}$ (Figure 10(b)). During the process, haptic feedback was provided. If the participant successfully grasped the PLA cylinder handed by the researcher within $5 \mathrm{~s}$ and could hold the PLA cylinder without dropping it for $10 \mathrm{~s}$, the trial was regarded as a success; otherwise, the trial was recorded as a failure.

In the pinch ability experiment, the participant was asked to control the thumb and the index finger of the soft robot hand to pinch a university ID card with a thickness of about $1 \mathrm{~mm}$ (Figure 10(c)). During the process, haptic feedback was provided. If the participant successfully pinched the card handed by the researcher within $5 \mathrm{~s}$ and could hold the card without dropping it for $10 \mathrm{~s}$, the trial was recognized as a success; otherwise, the trial was recorded as a failure.

\subsubsection{Experimental results}

The prototype of the soft robot hand was teleoperated to perform the nine motions (Figure 11). This activity demonstrated the potential of the soft robot hand performing complex tasks.

All participants had no experience in teleoperation before the experiment. Each experiment consisted of 60 (12 participants $\times 5$ repeats $)$ trials. The success rate of the force discrimination experiment was $100.00 \%$. In the grasp ability experiment, the success rate was $95.00 \%$, with three failed trials. In the pinch ability experiment, the success rate was $98.33 \%$, with one failed trial.

\subsection{Discussion}

According to the results of the characterization tests, the soft robot hand was slightly larger than the hand of an adult man. The soft robot hand prototype could satisfy 
This is an original manuscript / preprint of an article published by Taylor \& Francis in Advanced Robotics on 22 Sep 2020, available online:

https://www.tandfonline.com/doi/full/10.1080/01691864.2020.1822913.

$96.86 \%$ of the design requirements of the joint angles. Flexion and abduction forces of the hand prototype met the design requirements. These results proved that the soft robot hand exhibited adequate ROM and could generate sufficient force to perform most daily operational tasks. However, the MCPJ structure of the bending actuators should be further improved to achieve $100 \%$ of the ROM of the fingers.

In our design, pressure sensors were mounted on the fingertips of the soft robot hand to monitor the contact force. The sensors were smaller than the fingertips and thus the pressure sensors covered only a part of the fingertips. The contact force could only be measured when the sensor was within the contact area. An integral design of fingertips should be considered in our future studies to make sure the contact force would transfer to the effective range of the force sensor.

The haptic feedback actuators were small and lightweight. Wearing the haptic feedback actuators would not affect the movement of the fingers or add burden to the fingers of the operator. The force generated by the haptic feedback actuator met the design requirements. In this study, only the normal contact force was sensed and fed back to the operator. A rich haptic experience should ideally be provided. To provide both lateral- and normal-force feedback, the actuator size, complexity of the actuator structure, and difficulty of control are expected to increase. A tradeoff exists between the compactness of the actuator structure and the DOFs of the feedback forces. Moreover, the current fabrication process limited the further miniaturization of the actuator. During the 3D printing process, the working surface of the haptic feedback actuator was facing down to ensure the quality of this surface. Since no support materials were used to print the chamber, the bottom of the actuator (facing up during printing) would sag for the first few layers when printing. In order to ensure that the sagging material did not touch the working surface and had very little influence on the 
This is an original manuscript / preprint of an article published by Taylor \&

Francis in Advanced Robotics on 22 Sep 2020, available online:

https://www.tandfonline.com/doi/full/10.1080/01691864.2020.1822913.

performance of the haptic actuator, a thick air chamber was required. What's more, the

bottom surface should not deform too much when the actuator was activated. Therefore, the bottom surface of the actuator was required to be much thicker than the working surface. To further improve the fabrication process and miniaturize the actuator, further study is required.

3D printed pneumatic actuators tend to have problems of air tightness and durability [20]. In this paper, we adopted a similar 3D printing method as used in Yap et al.'s study [20], which claimed that the durability of the actuators created through their method was better than the 3D-printed actuators developed by Peele et al. using Projection Stereolithography [21]. Moreover, we tried to further improve the durability by making a silicone layer of film wrapping around the actuators. The silicone layer could improve air tightness of the actuators, but it cannot completely solve the durability problem. Those problems should be further studied in the future.

The results of the teleoperation performance experiment indicate that the participants without teleoperation experience could control the soft robot hand to perform different motions. The high success rates of the fingertip force discrimination, grasp, and pinch ability experiments proved that the soft robot hand can potentially be applied in teleoperational tasks. In this study, a rigid palm was used in the soft robot hand. Further study is required to develop a soft palm to achieve flexion motion of palm to provide better grasp and pinch ability. Further study about teleoperation with this robot hand attached to a robot arm is also desired.

\section{Conclusions}

In this paper, a soft robot hand with high adaptability to the shape of objects and fingertip haptic feedback for teleoperation to perform complex work under 
This is an original manuscript / preprint of an article published by Taylor \& Francis in Advanced Robotics on 22 Sep 2020, available online: https://www.tandfonline.com/doi/full/10.1080/01691864.2020.1822913.

circumstances such as grasping fragile items, rescuing injured people, and interacting with patients remotely is proposed. The proposed soft robot hand can perform flexion/extension and abduction/adduction finger motions. The fingertip contact forces are fed back to the hand of the operator by using small and lightweight haptic feedback actuators. Characterization tests and teleoperation performance tests involving human participants are performed to prove the feasibility of the soft robot hand. The soft robot hand prototype completely meets the output force requirements and can reach $96.86 \%$ of the design requirements of the joint angles. The soft robot hand can be tele-operated to perform different kinds of commonly-used motions in daily operational tasks, including light tool grasp, medium wrap grasp, fixed hook, spherical grasp, power disk grasp, tripod grasp, palmar grasp, lateral pinch, and precision tip pinch. The success rates of the fingertip force discrimination, grasp, and pinch ability experiments were $100 \%, 95.00 \%$, and $98.33 \%$, respectively. These results prove that the soft robot hand with fingertip haptic feedback for teleoperation has the potential for application in performing complex teleoperational tasks.

We intend to improve the palm and fingertip structures of the soft robot hand to provide enhanced grasp and pinch abilities. Haptic feedback will also be improved to provide a richer experience. The robustness and life cycle of the soft robot hand will be investigated by conducting additional tests. Teleoperation using the proposed robot hand attached to a robot arm will also be conducted.

\section{Acknowledgment}

We thank the participants of the experiments.

\section{Funding details}

This work was partially funded by the National Natural Science Foundation of China under Grant [51975451]; RSE - NSFC Joint Project under Grant [51911530243]; the 
This is an original manuscript / preprint of an article published by Taylor \&

Francis in Advanced Robotics on 22 Sep 2020, available online:

https://www.tandfonline.com/doi/full/10.1080/01691864.2020.1822913.

China Postdoctoral Science Foundation under Grant [2019M653586]; the Natural

Science Foundation of Shaanxi Province of China under Grant [2019JQ-332]; the State

Key Laboratory of Robotics under Grant [2019-O08]; and the Fundamental Research

Funds for the Central Universities under Grant [xzy012019012].

\section{References}

1. Michael H. How Animals Move: An Integrative View. Science. 2000;25:100106.

2. Jacobsen S, Iversen E, Knutti D, et al., editors. Design of the Utah/MIT dextrous hand. Proceedings. 1986 IEEE International Conference on Robotics and Automation; 1986: IEEE.

3. Loucks C, Johnson V, Boissiere P, et al., editors. Modeling and control of the Stanford/JPL hand. Proceedings. 1987 IEEE International Conference on Robotics and Automation; 1987: IEEE.

4. Mouri T, Kawasaki H, Yoshikawa K, et al. Anthropomorphic robot hand: Gifu hand III. International Conference on Control, Automation and Systems. 2002:1288-1293.

5. Grebenstein M, Chalon M, Friedl W, et al. The hand of the DLR hand arm system: Designed for interaction. The International Journal of Robotics Research. 2012;31(13):1531-1555.

6. Mouri T, Endo T, Kawasaki H. Review of gifu hand and its application. Mechanics based design of structures and machines. 2011;39(2):210-228.

7. Zhang A, Malhotra M, Matsuoka Y, editors. Musical piano performance by the ACT Hand. 2011 IEEE International Conference on Robotics and Automation; 2011: IEEE.

8. Kochan A. Shadow delivers first hand. Industrial robot: an international journal. 2005;32(1):15-16.

9. Ciocarlie M, Hicks FM, Holmberg R, et al. The Velo gripper: A versatile singleactuator design for enveloping, parallel and fingertip grasps. The International Journal of Robotics Research. 2014;33(5):753-767.

10. Backus SB, Dollar AM. An adaptive three-fingered prismatic gripper with passive rotational joints. IEEE Robotics and Automation Letters. 2016;1(2):668675.

11. Catalano MG, Grioli G, Farnioli E, et al. Adaptive synergies for the design and control of the Pisa/IIT SoftHand. The International Journal of Robotics Research. 2014;33(5):768-782.

12. Piazza C, Della Santina C, Catalano M, et al., editors. Softhand pro-d: Matching dynamic content of natural user commands with hand embodiment for enhanced prosthesis control. 2016 IEEE International Conference on Robotics and Automation (ICRA); 2016: IEEE.

13. Dollar AM, Howe RD. A robust compliant grasper via shape deposition manufacturing. IEEE/ASME transactions on mechatronics. 2006;11(2):154-161.

14. Dollar AM, Howe RD. The highly adaptive SDM hand: Design and performance evaluation. The international journal of robotics research. 2010;29(5):585-597. 
This is an original manuscript / preprint of an article published by Taylor \& Francis in Advanced Robotics on 22 Sep 2020, available online:

https://www.tandfonline.com/doi/full/10.1080/01691864.2020.1822913.

15. Deimel R, Brock O. A novel type of compliant and underactuated robotic hand for dexterous grasping. The International Journal of Robotics Research. 2016;35(1-3):161-185.

16. Dameitry A, Tsukagoshi H, editors. Lightweight underactuated pneumatic fingers capable of grasping various objects. 2016 IEEE International Conference on Robotics and Automation (ICRA); 2016: IEEE.

17. Deimel R, Brock O, editors. A compliant hand based on a novel pneumatic actuator. 2013 IEEE International Conference on Robotics and Automation; 2013: IEEE.

18. Watanabe T, Yamazaki K, Yokokohji Y. Survey of robotic manipulation studies intending practical applications in real environments-object recognition, soft robot hand, and challenge program and benchmarking. Advanced Robotics. 2017;31(19-20):1114-1132.

19. Wang Y, Gregory C, Minor MA. Improving Mechanical Properties of Molded Silicone Rubber for Soft Robotics Through Fabric Compositing. Soft robotics. 2018;5(3):272-290.

20. Yap HK, Ng HY, Yeow C-H. High-Force Soft Printable Pneumatics for Soft Robotic Applications. Soft robotics. 2016;3(3):144-158.

21. Peele BN, Wallin TJ, Zhao H, et al. 3D printing antagonistic systems of artificial muscle using projection stereolithography. Bioinspiration \& Biomimetics. 2015;10(5):055003.

22. Homberg BS, Katzschmann RK, Dogar MR, et al., editors. Haptic identification of objects using a modular soft robotic gripper. 2015 IEEE/RSJ International Conference on Intelligent Robots and Systems (IROS); 2015: IEEE.

23. Culha U, Nurzaman S, Clemens F, et al. SVAS3: strain vector aided sensorization of soft structures. Sensors. 2014;14(7):12748-12770.

24. Rone WS, Ben-Tzvi P, editors. Multi-segment continuum robot shape estimation using passive cable displacement. 2013 IEEE International Symposium on Robotic and Sensors Environments (ROSE); 2013: IEEE.

25. Zhao H, O'Brien K, Li S, et al. Optoelectronically innervated soft prosthetic hand via stretchable optical waveguides. Science Robotics. 2016;1(1):eaai7529.

26. Park Y-L, Chen B-R, Wood RJ. Design and fabrication of soft artificial skin using embedded microchannels and liquid conductors. IEEE Sensors Journal. 2012;12(8):2711-2718.

27. Bouzit M, Burdea G, Popescu G, et al. The Rutgers Master II-new design forcefeedback glove. IEEE/ASME Transactions on mechatronics. 2002;7(2):256-263.

28. Pacchierotti C, Sinclair S, Solazzi M, et al. Wearable haptic systems for the fingertip and the hand: taxonomy, review, and perspectives. IEEE transactions on haptics. 2017;10(4):580-600.

29. Vogel J, Castellini C, van der Smagt P, editors. EMG-based teleoperation and manipulation with the DLR LWR-III. 2011 IEEE/RSJ International Conference on Intelligent Robots and Systems; 2011: IEEE.

30. Cui L, Cupcic U, Dai JS. An optimization approach to teleoperation of the thumb of a humanoid robot hand: Kinematic mapping and calibration. Journal of Mechanical Design. 2014;136(9):091005.

31. Lee K-S, Jung M-C. Ergonomic evaluation of biomechanical hand function. Safety and health at work. 2015;6(1):9-17.

32. Schwarz RJ, Taylor C. The anatomy and mechanics of the human hand. Artificial limbs. 1955;2(2):22-35. 
This is an original manuscript / preprint of an article published by Taylor \&

Francis in Advanced Robotics on 22 Sep 2020, available online:

https://www.tandfonline.com/doi/full/10.1080/01691864.2020.1822913.

33. Cutkosky MR. On grasp choice, grasp models, and the design of hands for manufacturing tasks. IEEE Transactions on robotics and automation. 1989;5(3):269-279.

34. Zheng JZ, De La Rosa S, Dollar AM, editors. An investigation of grasp type and frequency in daily household and machine shop tasks. 2011 IEEE International Conference on Robotics and Automation; 2011: IEEE.

35. Polygerinos P, Wang Z, Galloway KC, et al. Soft robotic glove for combined assistance and at-home rehabilitation. Robotics and Autonomous Systems. 2015;73:135-143.

36. Van Zwieten KJ, Schmidt KP, Bex GJ, et al. An analytical expression for the DIP-PIP flexion interdependence in human fingers. 2015.

37. Massimino MJ, Sheridan TB. Teleoperator performance with varying force and visual feedback. Human factors. 1994;36(1):145-157.

38. Moody L, Baber C, Arvanitis TN. Objective surgical performance evaluation based on haptic feedback. Studies in health technology and informatics. 2002:304-310.

39. Pacchierotti C, Chinello F, Malvezzi M, et al., editors. Two finger grasping simulation with cutaneous and kinesthetic force feedback. International Conference on Human Haptic Sensing and Touch Enabled Computer Applications; 2012: Springer.

40. Pacchierotti C, Meli L, Chinello F, et al. Cutaneous haptic feedback to ensure the stability of robotic teleoperation systems. The International Journal of Robotics Research. 2015;34(14):1773-1787.

41. Solazzi M, Frisoli A, Bergamasco M, editors. Design of a novel finger haptic interface for contact and orientation display. 2010 IEEE Haptics Symposium; 2010: IEEE.

42. Prattichizzo D, Chinello F, Pacchierotti C, et al. Towards wearability in fingertip haptics: a 3-dof wearable device for cutaneous force feedback. IEEE Transactions on Haptics. 2013;6(4):506-516.

43. Frediani G, Mazzei D, De Rossi DE, et al. Wearable wireless tactile display for virtual interactions with soft bodies. Frontiers in bioengineering and biotechnology. 2014;2:31.

44. Chinello F, Malvezzi M, Pacchierotti C, et al., editors. Design and development of a 3RRS wearable fingertip cutaneous device. 2015 IEEE International Conference on Advanced Intelligent Mechatronics (AIM); 2015: IEEE.

45. Matheus K, Dollar AM, editors. Benchmarking grasping and manipulation: Properties of the objects of daily living. 2010 IEEE/RSJ International Conference on Intelligent Robots and Systems; 2010: IEEE.

46. Farstad JMG, Netland $\emptyset$, Welo T. Surface friction of rapidly prototyped wheels from 3D-printed thermoplastic elastomers: An experimental study. Procedia CIRP. 2017;60:247-252.

Table 1. ROM of finger joints

\begin{tabular}{ll}
\hline Item & Angle( $($ ) \\
\hline Flexion ROM of MCPJ of thumb & 61.00 \\
Flexion ROM of IPJ of thumb & 81.00 \\
Flexion ROM of CMCJ of thumb & 49.00 \\
Flexion ROM of MCPJ of index finger & 80.00 \\
Flexion ROM of PIPJ of index finger & 104.00
\end{tabular}


This is an original manuscript / preprint of an article published by Taylor \& Francis in Advanced Robotics on 22 Sep 2020, available online: https://www.tandfonline.com/doi/full/10.1080/01691864.2020.1822913.

\begin{tabular}{ll} 
Flexion ROM of DIPJ of index finger & 68.00 \\
Flexion ROM of MCPJ of middle finger & 85.00 \\
Flexion ROM of PIPJ of middle finger & 107.00 \\
Flexion ROM of DIPJ of middle finger & 70.00 \\
Flexion ROM of MCPJ of ring finger & 87.00 \\
Flexion ROM of PIPJ of ring finger & 107.00 \\
Flexion ROM of DIPJ of ring finger & 66.00 \\
Flexion ROM of MCPJ of pinky finger & 86.00 \\
Flexion ROM of PIPJ of pinky finger & 104.00 \\
Flexion ROM of DIPJ of pinky finger & 69.00 \\
Abduction ROM between thumb and index finger & 57.00 \\
Abduction ROM between index finger and middle finger & 28.00 \\
Abduction ROM between middle finger and ring finger & 21.00 \\
Abduction ROM between ring finger and pinky finger & 32.00 \\
\hline
\end{tabular}

Table 2. Parameter adjustments to Lulzbot TAZ 6

\begin{tabular}{ll}
\hline Printing parameters & Value \\
\hline Lay Height & $0.1 \mathrm{~mm}$ \\
Initial Lay Height & $0.5 \mathrm{~mm}$ \\
Line Width & $0.5 \mathrm{~mm}$ \\
Wall Thickness & $1.0 \mathrm{~mm}$ \\
Infill Density & $70 \%$ \\
Infill Overlap Percentage & $20 \%$ \\
Printing Temperature & $230{ }^{\circ} \mathrm{C}$ \\
Initial Printing Temperature & $230{ }^{\circ} \mathrm{C}$ \\
Final Printing Temperature & $230{ }^{\circ} \mathrm{C}$ \\
\hline
\end{tabular}

Table 3. Comparison between the ROM of the soft robot hand and the design requirements

\begin{tabular}{llll}
\hline Item & $\begin{array}{l}\text { Achieved } \\
\text { angle }(\circ)\end{array}$ & $\begin{array}{l}\text { Requirement } \\
(\circ)\end{array}$ & Percentage \\
\hline Flexion of thumb MCPJ & 66.25 & 61.00 & $100.00 \%$ \\
Flexion of thumb IPJ & 85.30 & 81.00 & $100.00 \%$ \\
Flexion of thumb CMCJ of thumb & 64.00 & 49.00 & $100.00 \%$ \\
Flexion of index finger MCPJ & 71.10 & 80.00 & $88.88 \%$ \\
Flexion of index finger PIPJ & 110.31 & 104.00 & $100.00 \%$ \\
Flexion of index finger DIPJ & 94.70 & 68.00 & $100.00 \%$ \\
Flexion of middle finger MCPJ & 74.42 & 85.00 & $87.55 \%$ \\
Flexion of middle finger PIPJ & 100.19 & 107.00 & $93.64 \%$ \\
Flexion of midder finger DIPJ & 86.76 & 70.00 & $100.00 \%$ \\
Flexion of ring finger MCPJ & 75.88 & 87.00 & $87.22 \%$ \\
Flexion of ring finger PIPJ & 106.59 & 107.00 & $99.62 \%$ \\
Flexion of ring finger DIPJ & 90.20 & 66.00 & $100.00 \%$ \\
Flexion of pinky MCPJ & 71.70 & 86.00 & $83.37 \%$ \\
Flexion of pinky PIPJ & 107.15 & 104.00 & $100.00 \%$ \\
Flexion of pinky DIPJ & 82.22 & 69.00 & $100.00 \%$
\end{tabular}


This is an original manuscript / preprint of an article published by Taylor \& Francis in Advanced Robotics on 22 Sep 2020, available online:

https://www.tandfonline.com/doi/full/10.1080/01691864.2020.1822913.

Abduction between thumb and index finger

Abduction between index finger and middle finger

28.00

$100.00 \%$

Abduction between middle finger and ring finger

21.00

$100.00 \%$

Abduction between ring finger and pinky finger

Table 4. Forces required to unfold the activated soft robot hand fingers and the friction force of each finger

\begin{tabular}{lll}
\hline Finger & Force $(\mathbf{N})$ & Friction force $(\mathbf{N})$ \\
\hline Thumb & $13.71 \pm 1.02$ & 8.36 \\
Index finger & $16.49 \pm 0.63$ & 10.06 \\
Middle finger & $15.10 \pm 0.10$ & 9.21 \\
Ring finger & $12.97 \pm 0.19$ & 7.91 \\
Pinky finger & $15.07 \pm 0.97$ & 9.19 \\
\hline
\end{tabular}

Soft robot hand with flexion and pressure sensor
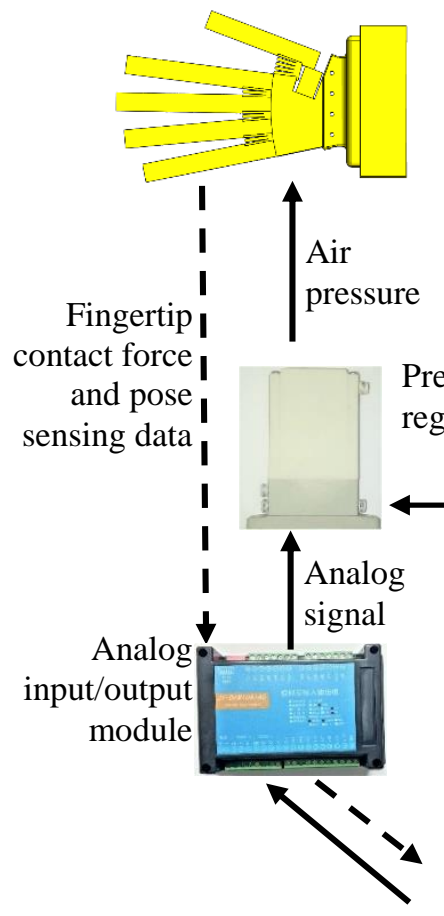

Haptic feedback actuators

Pressure regulator

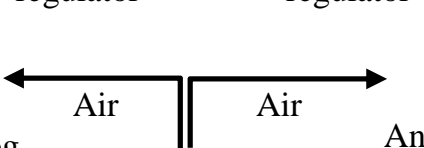

Analog

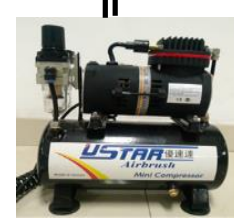

Air compressor

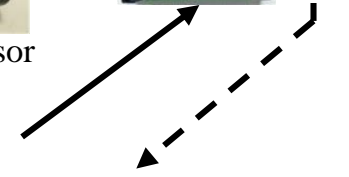

Analog

input/output

module

Figure 1. Diagrammatic sketch of a soft robot hand with fingertip haptic feedback for teleoperation. 
This is an original manuscript / preprint of an article published by Taylor \& Francis in Advanced Robotics on 22 Sep 2020, available online: https://www.tandfonline.com/doi/full/10.1080/01691864.2020.1822913.

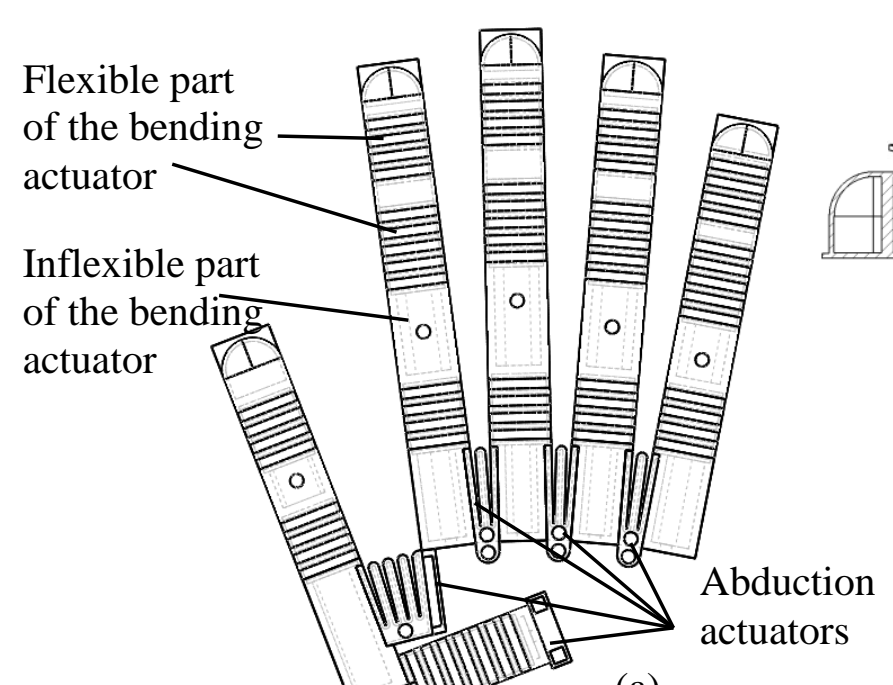

(a)

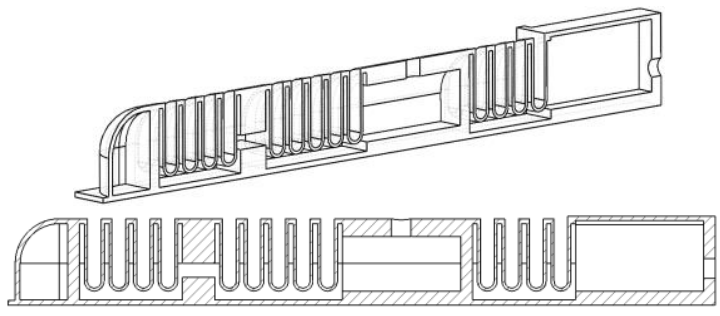

(b)

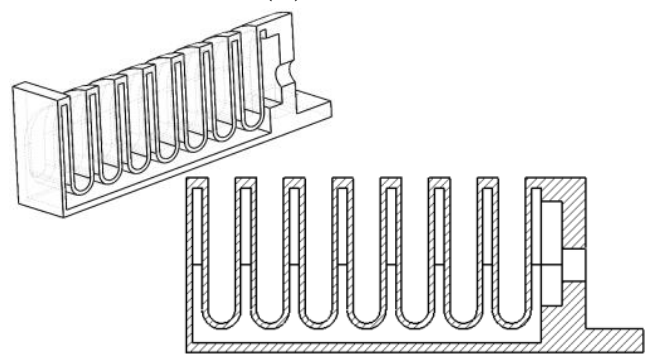

(c)

Figure 2. The 3D models of hand actuators: (a) the assembly drawing; (b) the longitudinal section of the index finger; and (c) the longitudinal section of the CMCJ actuator.

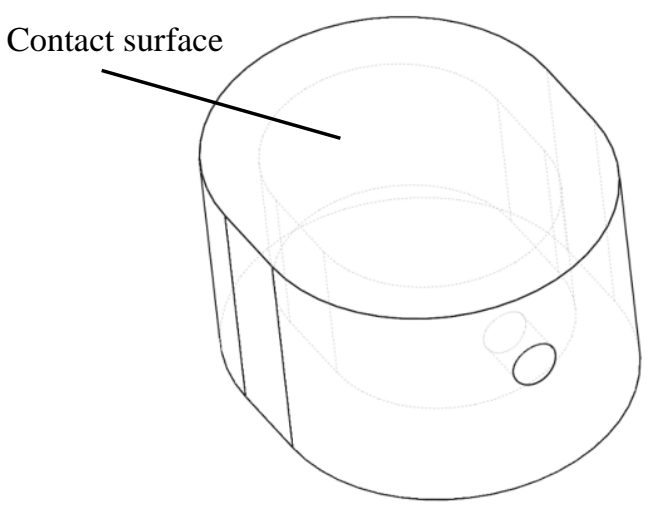

(a)

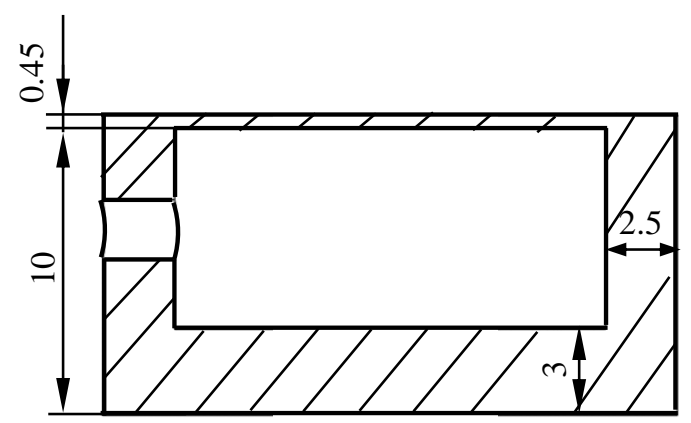

(b)

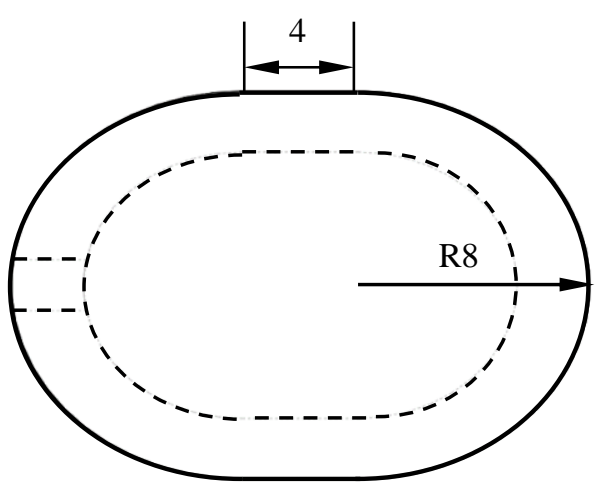

(c)

Figure 3. The haptic feedback actuator: (a) 3D model, (b) the cross section, and (c) the top view. 
This is an original manuscript / preprint of an article published by Taylor \& Francis in Advanced Robotics on 22 Sep 2020, available online: https://www.tandfonline.com/doi/full/10.1080/01691864.2020.1822913.

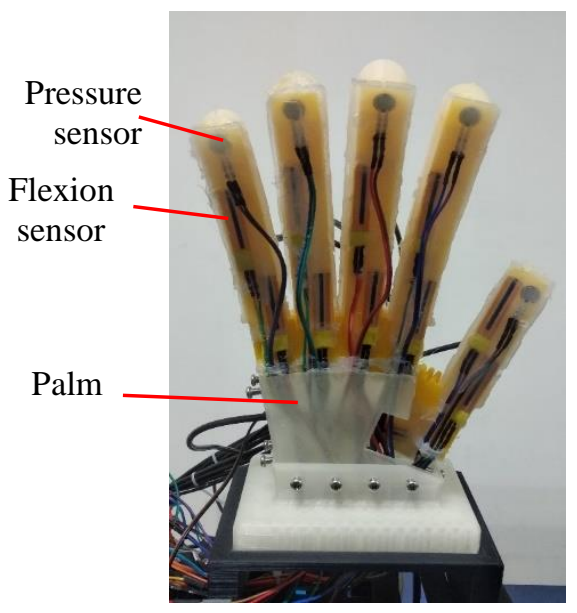

(a)

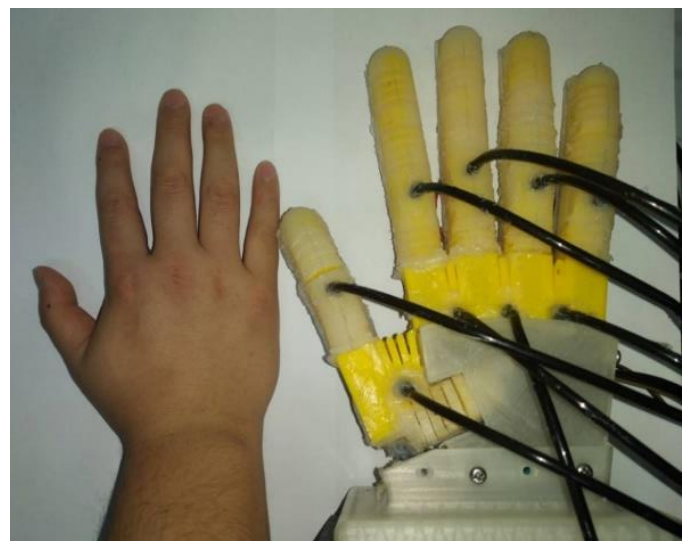

(b)

Haptic feedback actuators

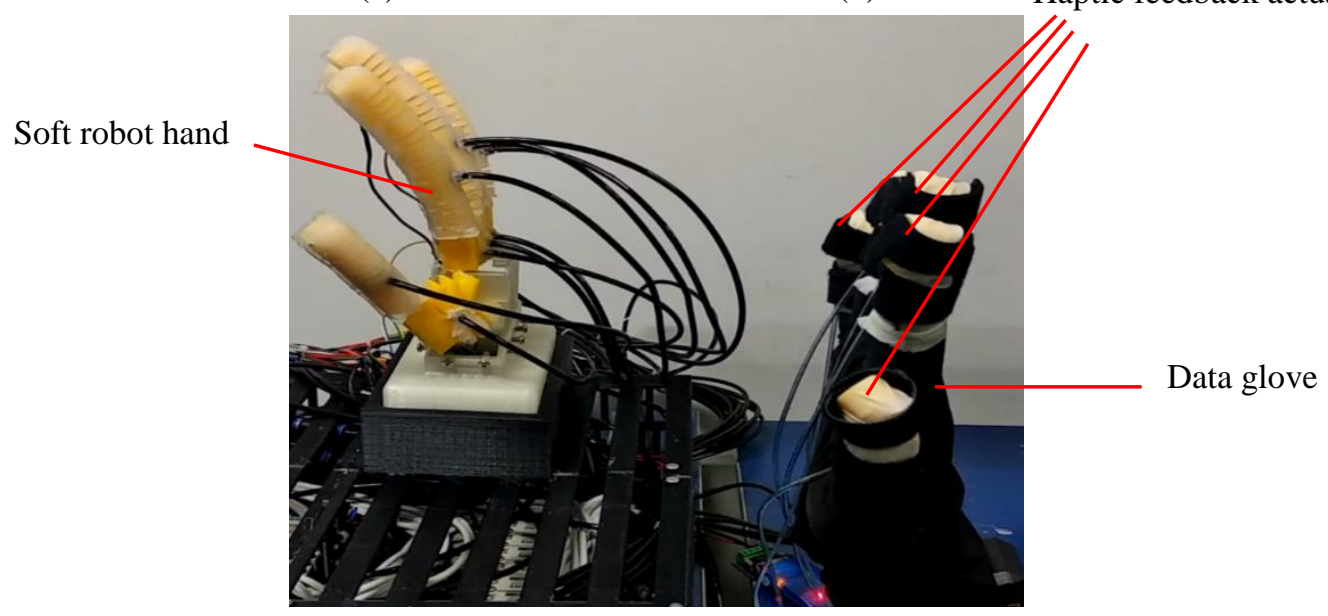

(c)

Figure 4. The soft robot hand: (a) palm side, (b) back side, and (c) soft robot hand with fingertip haptic feedback for teleoperation.

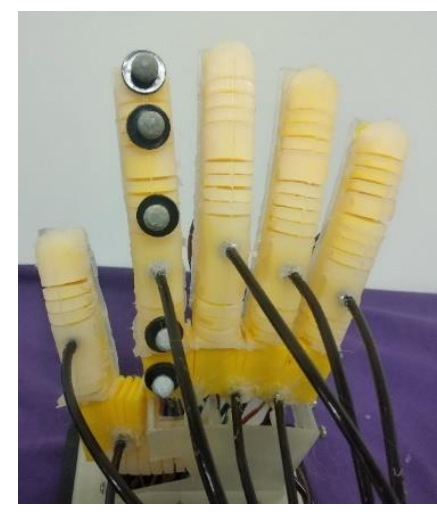

(a)

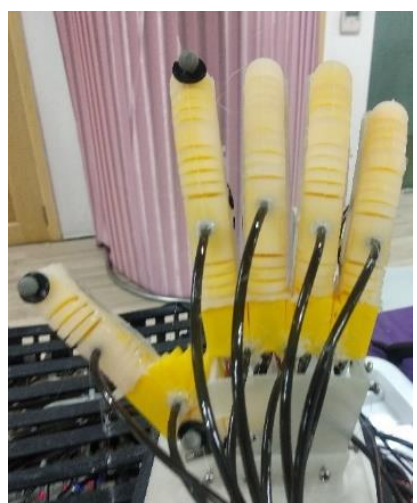

(b)

Figure 5. The infrared marker ball position on the soft robot hand: (a) the flexion angle test and (b) the abduction angle test. 
This is an original manuscript / preprint of an article published by Taylor \&

Francis in Advanced Robotics on 22 Sep 2020, available online:

https://www.tandfonline.com/doi/full/10.1080/01691864.2020.1822913.

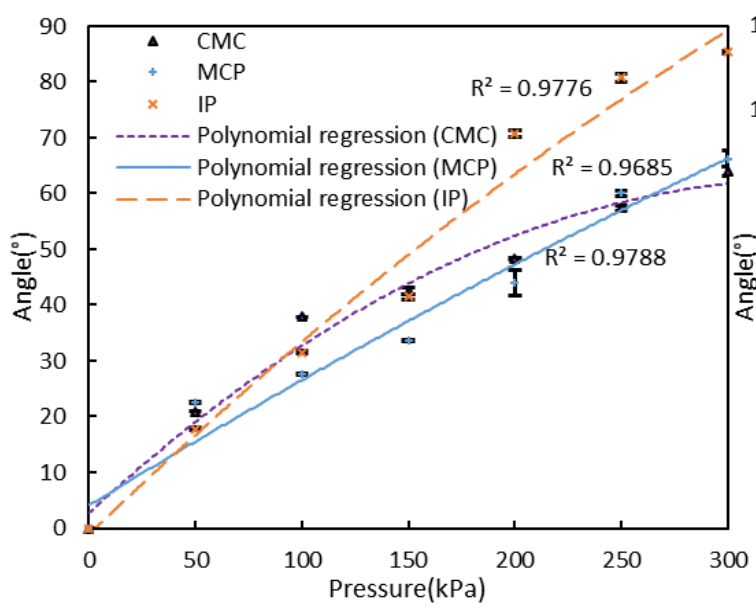

(a)

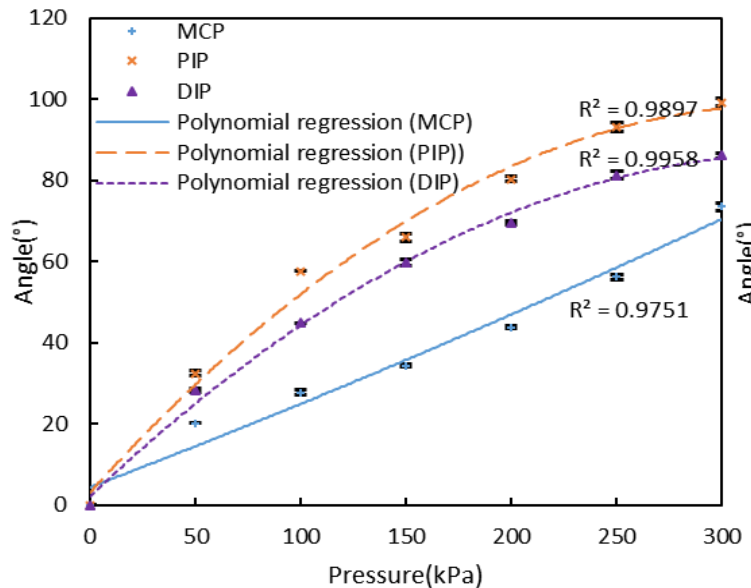

(c)

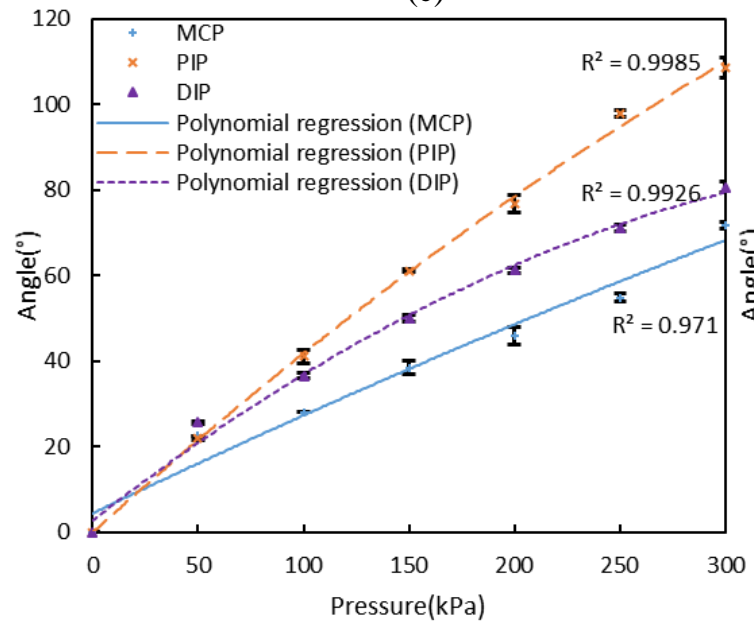

(e)

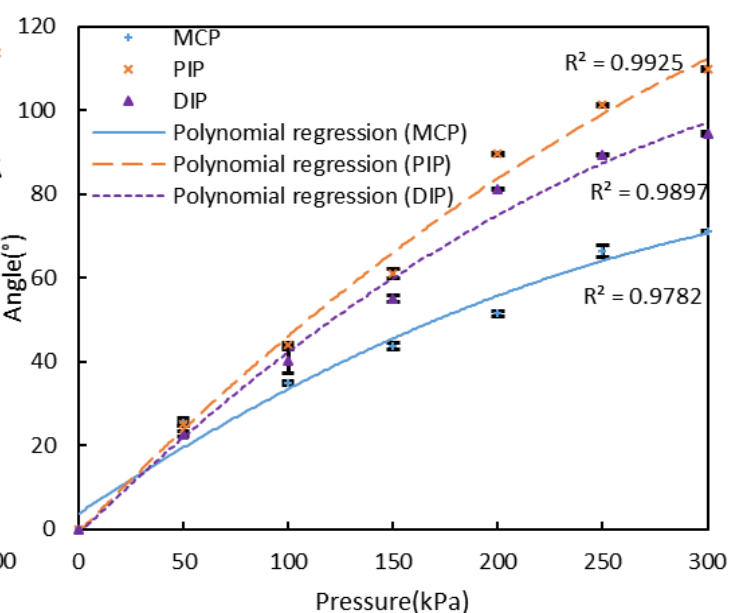

(b)

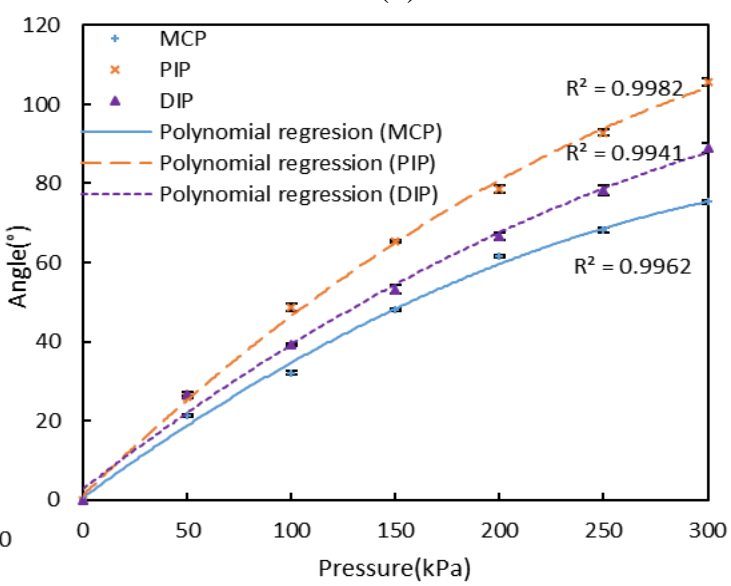

(d)

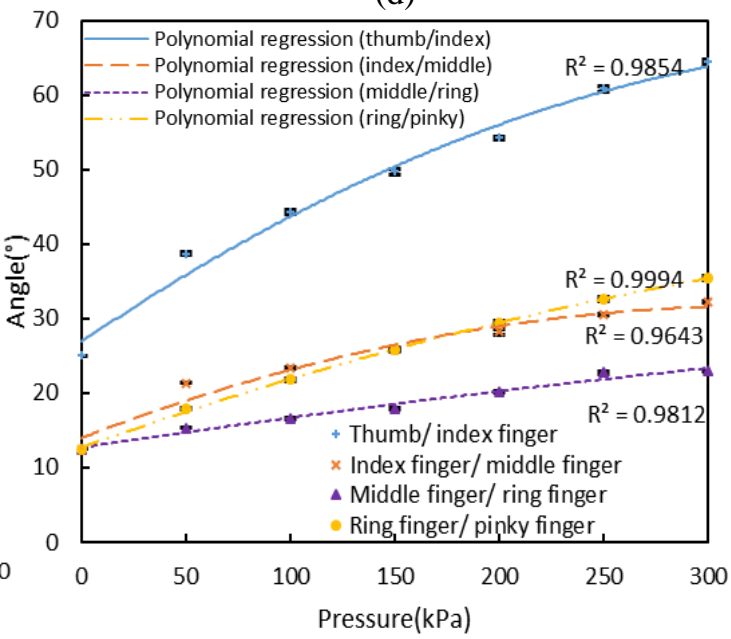

(f)

Figure 6. The average flexion and abduction angles of soft robot hand: (a) flexion angles of thumb, (b) flexion angles of index finger, (c) flexion angles of middle finger, (d) flexion angles of ring finger, (e) flexion angles of pinky finger, and (f) abduction angles. 
This is an original manuscript / preprint of an article published by Taylor \& Francis in Advanced Robotics on 22 Sep 2020, available online: https://www.tandfonline.com/doi/full/10.1080/01691864.2020.1822913.

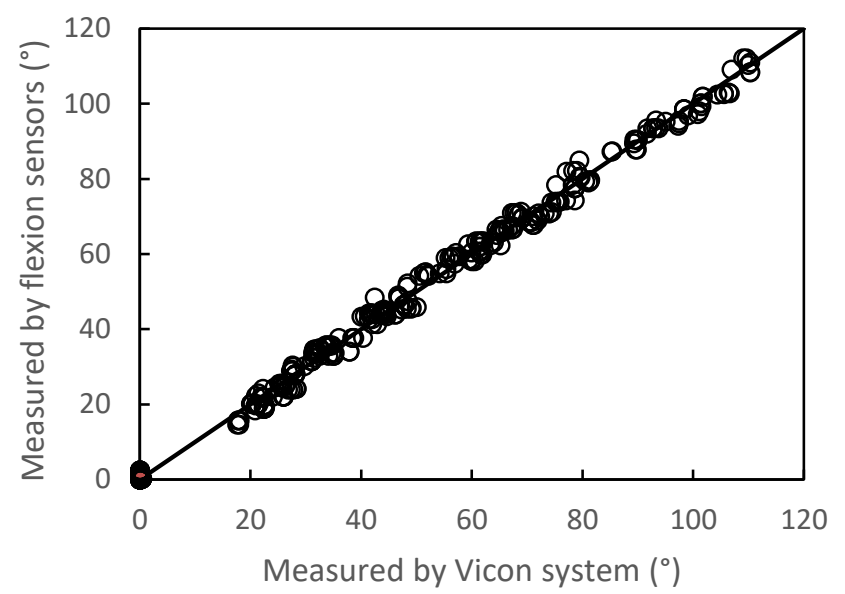

Figure 7. The outputs from the flexion sensors vs. the outputs from a VICON motion capture system during the pose sensing evaluation test. 
This is an original manuscript / preprint of an article published by Taylor \& Francis in Advanced Robotics on 22 Sep 2020, available online: https://www.tandfonline.com/doi/full/10.1080/01691864.2020.1822913.

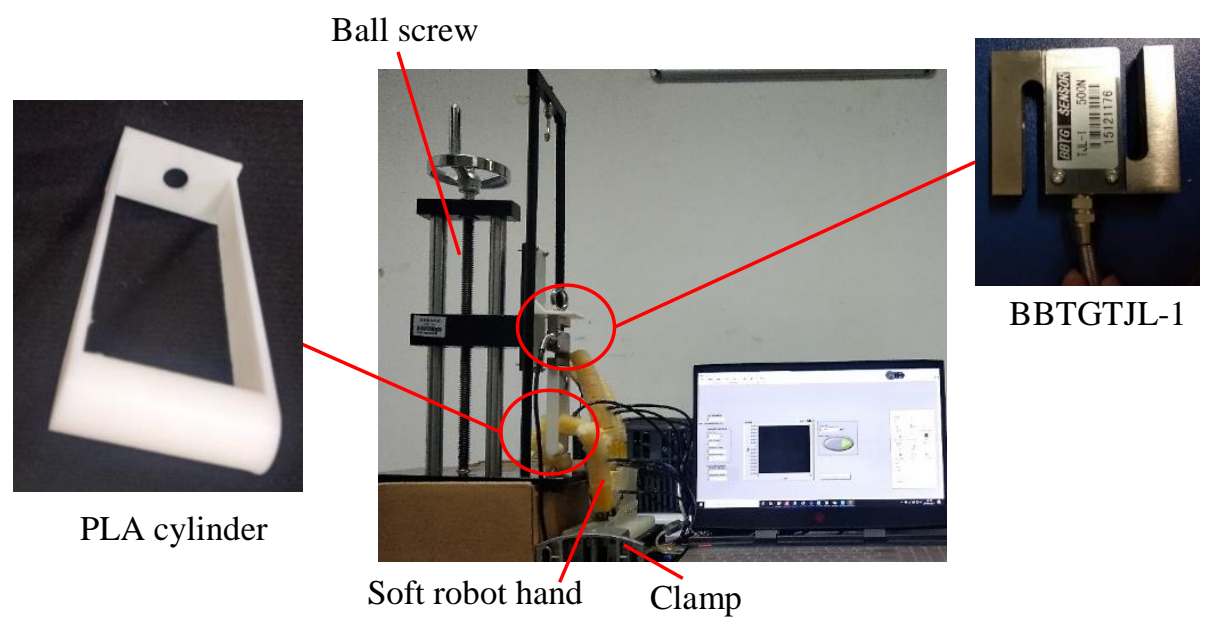

(a)

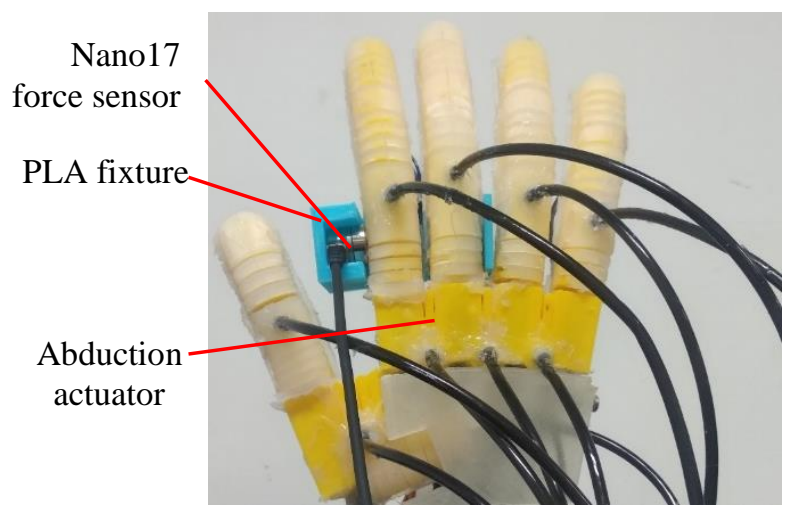

(b)

Figure 8. Experimental set-up for (a) the flexion output force test and (b) the abduction output force of soft robot hand fingers. 
This is an original manuscript / preprint of an article published by Taylor \& Francis in Advanced Robotics on 22 Sep 2020, available online: https://www.tandfonline.com/doi/full/10.1080/01691864.2020.1822913.

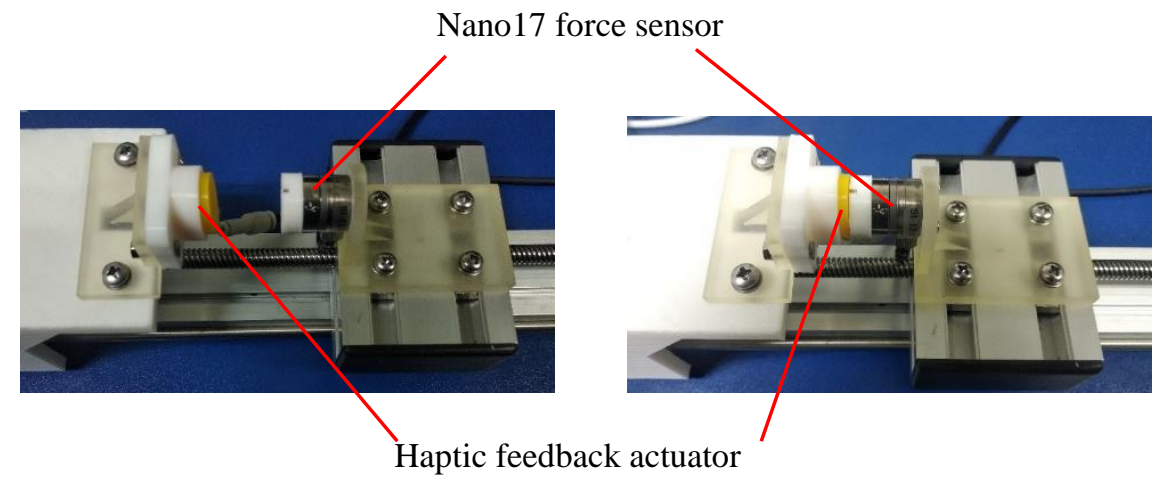

(a)

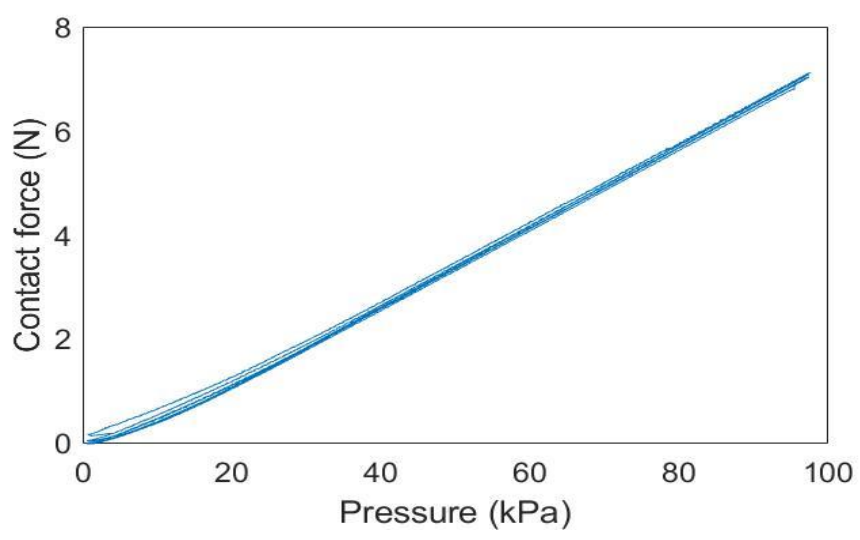

(b)

Figure 9. The output force test of fingertip haptic feedback actuators: (a) experimental set-up and (b) output force results. 
This is an original manuscript / preprint of an article published by Taylor \& Francis in Advanced Robotics on 22 Sep 2020, available online: https://www.tandfonline.com/doi/full/10.1080/01691864.2020.1822913.

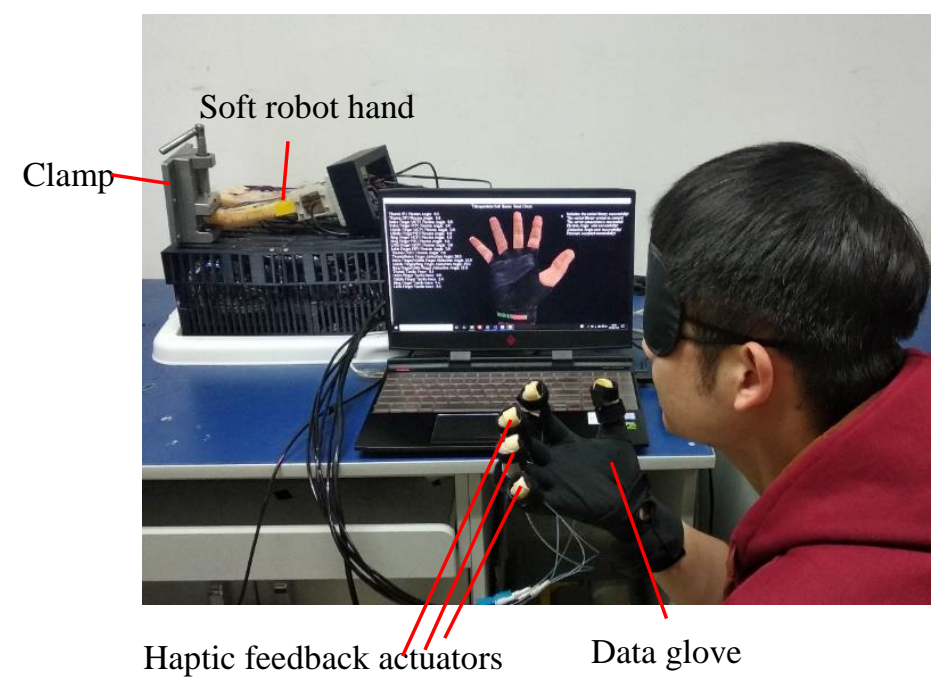

(a)

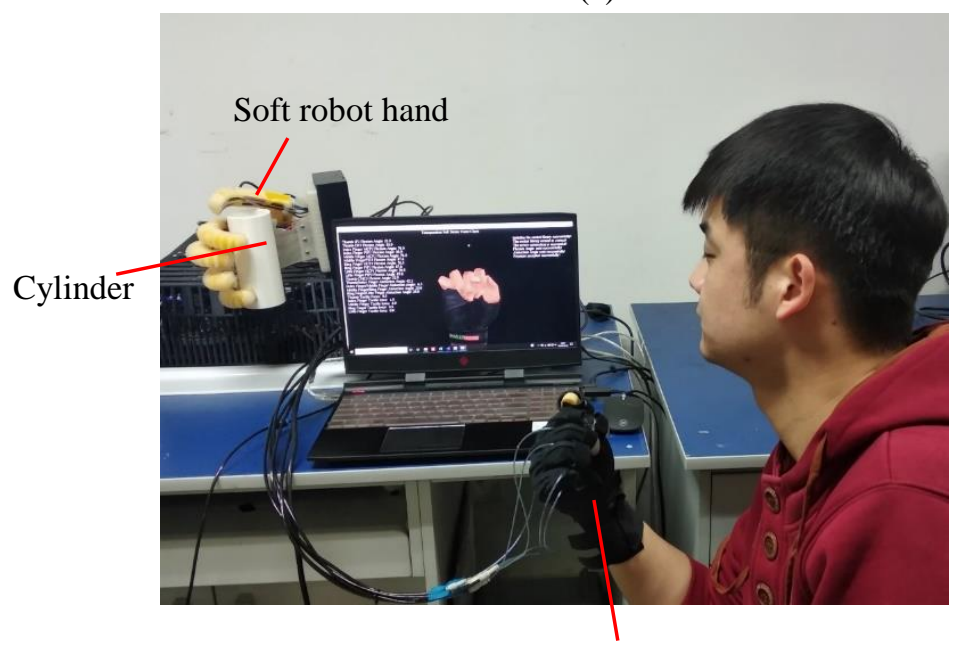

Data glove

(b)

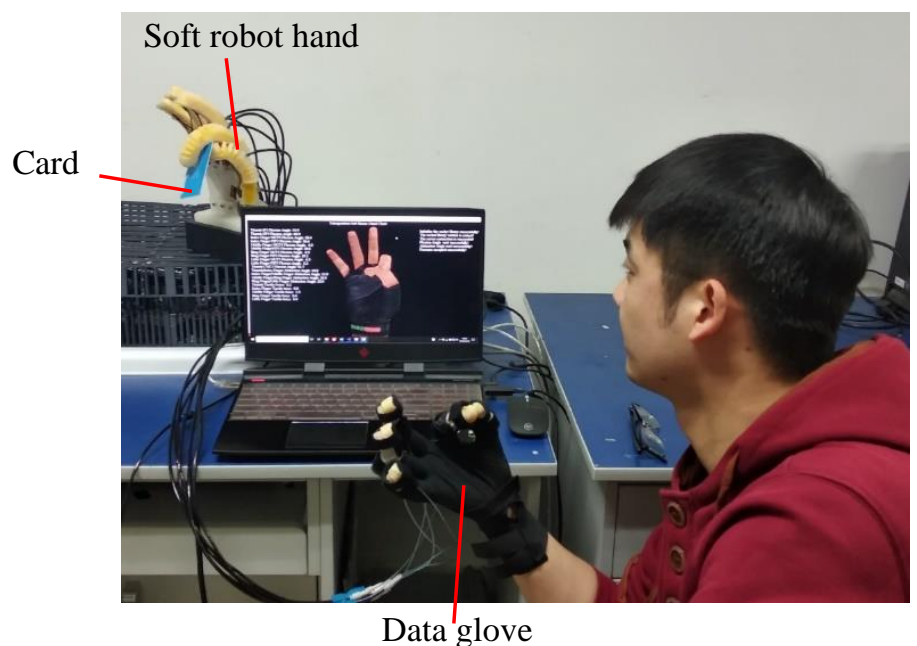

(c) 
This is an original manuscript / preprint of an article published by Taylor \& Francis in Advanced Robotics on 22 Sep 2020, available online: https://www.tandfonline.com/doi/full/10.1080/01691864.2020.1822913.

Figure 10. Experimental set-up of (a) fingertip force discrimination, (b) grasp ability, and (c) pinch ability.

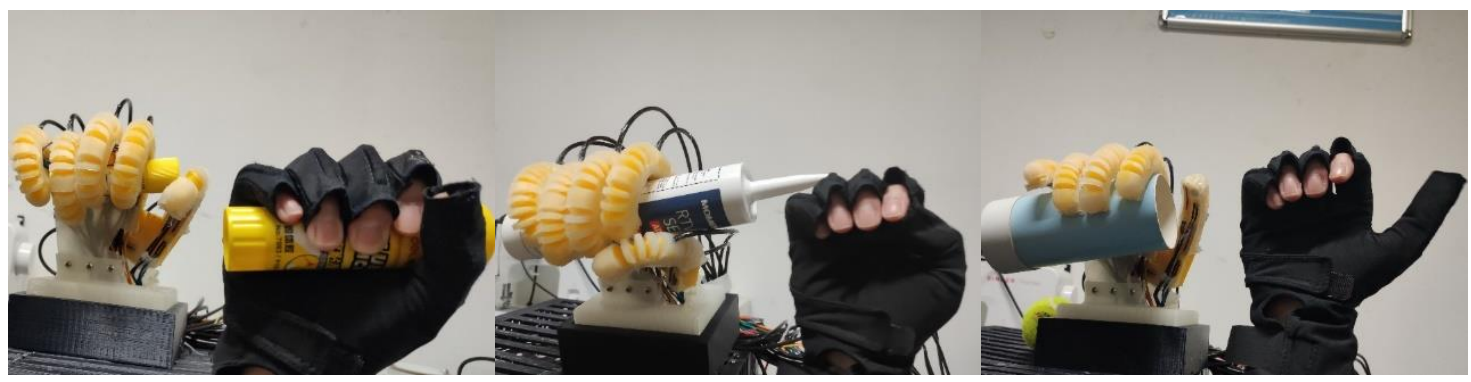

(a)

(b)

(c)

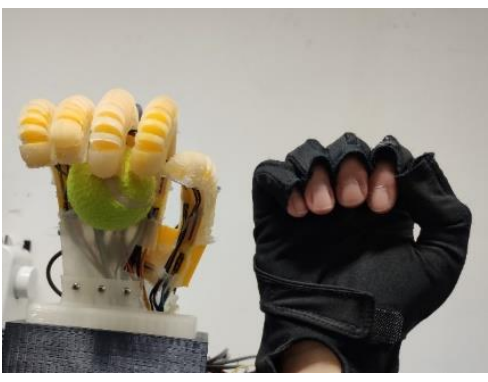

(d)

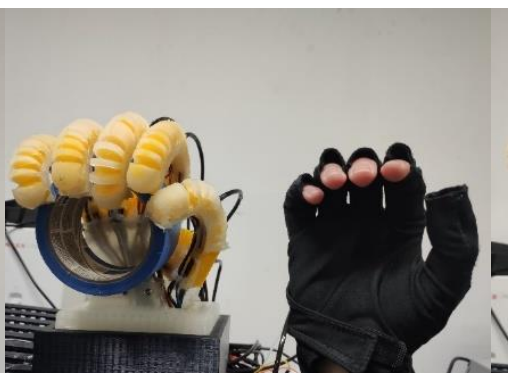

(e)

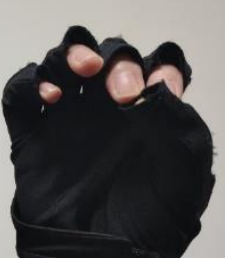

(f)

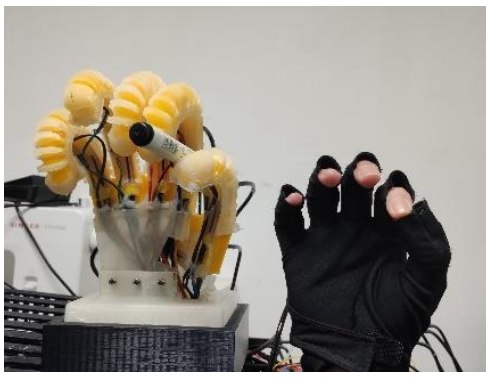

(g)

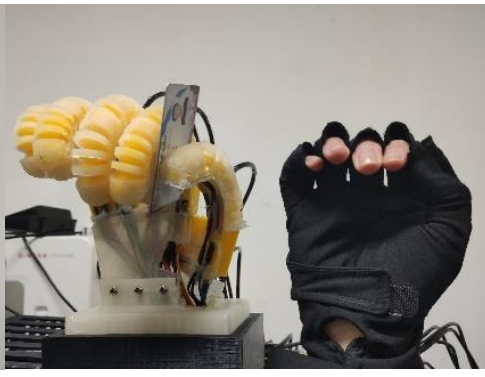

(h)

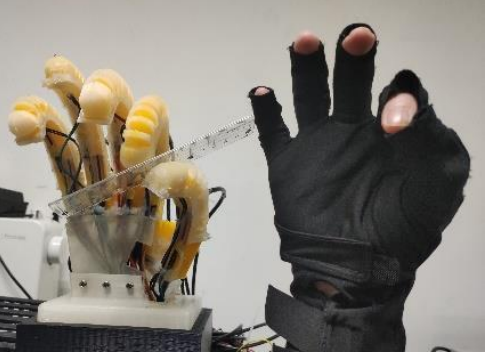

(i)

Figure 11. The experiment motions: (a) light tool grasp, (b) medium wrap grasp, (c) fixed hook, (d) spherical grasp, (e) power disk grasp, (f) tripod grasp, (g) palmar grasp, (h) lateral pinch, and (i) precision tip pinch. 